\title{
Erratum to: Algorithms for the Orthographic-n-Point Problem
}

\author{
Carsten Steger $^{1}$ (D)
}

Published online: 5 October 2017

(C) Springer Science+Business Media, LLC 2017

\section{Erratum to: J Math Imaging Vis}

\section{DOI 10.1007/s10851-017-0756-y}

The original version of this article contained references to step numbers in some of the algorithms that were incorrect.

The original article has been corrected.

The online version of the original article can be found under https:// doi.org/10.1007/s10851-017-0756-y.

\section{Carsten Steger}

steger@mvtec.com

1 MVTec Software GmbH, Arnulfstraße 205, 80634 München, Germany 